J. Lake Sci. (湖泊科学), 2006, 18(6):655-660

http:// www. jlakes. org. E-mail: jlakes@ niglas. ac.cn

(c) 2006 by Journal of Lake Sciences

\title{
山东省三个鲤鱼群体遗传多样性及亲缘关系的微卫星标记分析
}

\author{
马洪雨 ${ }^{1}$, 岳永生 ${ }^{1}$, 郭金峰 $^{1}$, 公维华 ${ }^{2}$, 王 慧 ${ }^{1 * *}$ \\ ( 1 : 山东农业大学动物分子遗传育种实验室,泰安 271018) \\ (2: 中国农业科学院畜牧研究所,北京 100094)
}

摘 要: 利用微卫星标记技术对 3 个鲤鱼群体 (DP、WS 和 SY) 的遗传多样性及亲缘关系进行研究. 结果表明, 3 个群体的 遗传多样性总体水平较高, 其中 SY 群体最高, WS 群体次之, DP 群体最低. 根据基因型频率 $(P)$ 检验了各位点的 Hardy Weinberg 平衡情况. Fis 值表明 3 个群体中共有 32 个微卫星位点的杂合度观测值过剩. 3 个群体间, WS 和 SY 群体间的遗 传相似系数最大 $(0.8320)$, 遗传距离最小 $(0.1680)$, 表明这两个群体亲缘关系较近; WS 和 DP 群体间的遗传相似系数最 小 $(0.8288)$, 遗传距离最大 $(0.1712)$, 表明这两个群体亲缘关系较远.

关键词:鲤鱼;微卫星;遗传多样性;亲缘关系

\section{Microsatellite marker analysis of genetic diversity and phylogenetic relationships in three populations of Cyprinus carpio L., Shandong Province}

\author{
MA Hongyu ${ }^{1}$, YUE Yongsheng ${ }^{1}$, GUO Jinfeng ${ }^{1}$, GONG Weihua ${ }^{2} \&$ WANG Hui ${ }^{1}{ }^{*}$ \\ (1: Molecular genetics and animal breeding Lab, Shandong Agricultural University, Taían 271018, P. R. China) \\ (2: Institute of Animal Science, Chinese Academy of Agricultural Sciences, Beijing 100094, P. R. China)
}

\begin{abstract}
The genetic diversity and the phylogenetic relationships of three Cyprinus carpio L. populations were studied by using microsatellite marker technology. It showed that the genetic diversity level of three Cyprinus carpio L. populations was high. population was the highest, WS population was lower, and DP population was the lowest. In order to check the depature of microsatellite loci from $\mathrm{HWE}$, the genotypic frequencies were calculated. Furthermore, 32 cases of observed heterozygosity excess $($ Fis $<0)$ were observed in three populations. The genetic similarity index between WS population and SY population was the highest $(0.8320)$ among the three populations, and their genetic distance was the lowest $(0.1680)$, which indicated that the phylogenetic relationship between these two populations was quite close. On the other hand, the genetic similarity index between WS population and DP population was the lowest $(0.8288)$, and their genetic distance was the highest $(0.1712)$, which indicated that the phylogenetic relationship between these two populations was quite far.
\end{abstract}

Key words : Cyprinus carpio L. ; microsatellite; genetic diversity; phylogenetic relationship

鲤鱼是中国最重要的淡水经济鱼类,品种繁多,广泛分布于中国各大水系中. 近十几年来,科研工作者 利用常规育种和雌核发育等技术,已经成功培育出了荷包红鲤、高寒鲤、建鲤等多个品系, 它们在生长速度、 抗逆性等方面均优于亲本 ${ }^{[1-3]}$. 到目前为止, 黑龙江野鲤、荷包红鲤、高寒鲤、黄河鲤、长江鲤、“江西三红” 的群体遗传结构及遗传多样性研究已有报道 ${ }^{[4-6]}$,均显示了相对稳定的群体遗传结构和丰富的遗传多样性 水平.

东平湖地处黄河下游,担负着汛期黄河泄洪的任务;微山湖通过京杭大运河与长江相通,两个湖区的鲤 鱼群体受到了外来群体 (黄河鲤、长江鲤) 的冲击,品质资源有所改变.近年来,两个湖区蓄水调节能力持续

* 资金项目: 国家科技部资助项目 (04033810) 和山东省科技厅资助项目 (04038335) 共同资助. $2005-11-28$ 收稿; 2006-03-07 收修改稿.马洪雨,男,1979 生,硕士研究生; E-mail: mhyxl@ 163.com.

** 通讯作者;E-mail: wanghui 2328@163.com. 
下降、过度捕捞与环境污染等情况不断加剧, 导致天然野生鲤鱼群体数量急剧下降. 泰安市水产研究所是山 东省及周边地区重要的鲤鱼良种供应基地, 苗种品质的优劣直接影响着广大养殖户的经济利益. 因此, 充分 了解山东省具有代表性的 3 个鲤鱼群体的种群结构、群体遗传多样性水平、生活环境状况,对进行种质资源 保护性开发利用,以及提高周边老百姓的生活水平具有重要意义.

微卫星标记与其它的遗传标记 (同工酶、RAPD 等) 相比, 具有较高的突变率 ${ }^{[7,8]}$, 因而在许多物种中显 示出较高水平的遗传变异 ${ }^{[9]}$, 被广泛应用于遗传作图 ${ }^{[10]}$ 、亲子鉴定 ${ }^{[11]}$ 及群体遗传多样性分析 ${ }^{[12,13]}$ 等研究 工作中. 关于微山湖鲤鱼生理生化方面的研究已有报道 ${ }^{[14]}$, 尚未见微卫星 DNA 标记在 3 个鲤鱼群体中应 用的报道. 本文利用微卫星标记技术, 采用 26 对普通鲤鱼微卫星引物对山东省具有代表性的 3 个鲤鱼群体 的遗传结构及亲缘关系进行了研究.

\section{1 材料与方法}

\section{1 材料}

鲤鱼 (Cyprinus carpio L.) )三个群体共 95 尾. 其中 30 尾取自东平湖 ( 以下简称“DP”) ;35 尾取自微山湖 (以下简称“WS”);30 尾取自泰安市水产研究所 (以下简称“SY”).

\section{2 基因组 DNA 提取}

提取方法见参考文献 ${ }^{[15]}$.

\subsection{PCR 反应与产物检测}

PCR 反应体系: DNA 模板 $0.7 \mu \mathrm{l}$; 上下游引物各 $0.6 \mu \mathrm{mol} / \mathrm{L}$; [ $\left.\mathrm{Mg}^{2+}\right]$ 见表 1; [ dNTPs $] 200 \mu \mathrm{mol} / \mathrm{L}$; Taq DNA 聚合酶 $0.625 \mathrm{U} ; 10 \times$ PCR buffer $2.5 \mu \mathrm{l}$; 补充灭菌双蒸水至终体积 $25 \mu \mathrm{L}$. PCR 反应程序: $94^{\circ} \mathrm{C}$ 预变性 $4 \mathrm{~min}, 94^{\circ} \mathrm{C}$ 变性 $30 \mathrm{~s}$, 退火 (表 1) $50 \mathrm{~s}, 72{ }^{\circ} \mathrm{C}$ 延伸 $50 \mathrm{~s}, 30$ 个循环; 再于 $72^{\circ} \mathrm{C}$ 延伸 $7 \mathrm{~min}$. 扩增产物用 $12 \%$ 的非变 性聚丙烯酰胺凝胶电泳检测, 银染, 数码相机拍照保存.

表 1 微卫星位点的特征

Tab. 1 Characteristics of microsatellete loci

\begin{tabular}{|c|c|c|c|c|}
\hline 微卫星位点 & 上游引物序列 & 下游引物序列 & 退火温度 $\left({ }^{\circ} \mathrm{C}\right)$ & $\mathrm{Mg}^{2+}$ 浓度 \\
\hline HLJ010 & TAGTGGGCACTGCAACTGTC C & ZATTCATTGTCATTTTGAGAAAGG & 52 & 1.7 \\
\hline HLJ011 & TTAGCCAGCCAGAGACAAGC & CACTGCCACAAACCCATCTA & 56 & 1.7 \\
\hline HLJ013 & AAAAAGGCTGAAGCAGCAAT & GATCAGCCATGTTCAAGGAAC & 52 & 1.7 \\
\hline MFW 1 & GTCCAGACTGTCATCAGGAG & GAGGTGTACACTGAGTCACGC & 55 & 1.5 \\
\hline MFW3 & GATCAGAAGGTACAGAGAAG & CCTTACAGAAAACCTGTTTGC & 55 & 1.5 \\
\hline MFW4 & TCCAAGTCAGTTTAATCACCG & GGGAAGCGTTGACAACAAGC & 57 & 1.7 \\
\hline MFW6 & ACCTGATCAATCCCTGGCTC & TTGGGACTTTTAAATCACGTTG & 57 & 1.7 \\
\hline MFW 13 & ATGATGAGAACATTGTTTACAG & TGAGAGAACAATGTGGATGAC & 55 & 1.7 \\
\hline MFW 14 & CAGAAGCTTCTGGAAATCTGAG & GCGAGAAGATTGATGGACAAC & 53 & 1.7 \\
\hline MFW 16 & GTCCATTGTGTCAAGATAGAG & TCTTCATTTCAGGCTGGAAAG & 55 & 2.0 \\
\hline MFW 18 & GTCCCTGGTAGTGAGTGAGT & GCGTTGACTTGTTTTATACTAG & 55 & 1.7 \\
\hline MFW 19 & GAATCCTCCATCATGCAAAC & GCACAAACTCCACATTGTGCC & 55 & 1.7 \\
\hline MFW30 & GGTCAACAAGTAGTTGTGCAG & CCATCTCTGTCATTGCAACAG & 57 & 1.7 \\
\hline
\end{tabular}

\section{4 数据处理}

根据每个个体产生的条带位置, 利用 LabImage (Version 2.7.2) 软件辅助确定基因型并计算等位基因大 小; 利用 POPGENE32 (Version 1.31) 软件计算等位基因频率 $(P)$ 、多态信息含量 $(P I C)$ 、基因杂合度 $(H) 、 F i s$ 值、Shannon 多样性指数并对各位点进行 Hardy - Weinberg 平衡检测 ; 计算各群体间的遗传相似性指数和遗 
传距离, 采用非加权配对算术平均法 (UPGMA) 构建 3 个鲤鱼群体的系统发生树.

\section{2 结果}

\section{1 等位基因组成}

3 个群体在 13 个微卫星位点上共检测出 97 个等位基因,每个群体的平均等位基因数分别为 $6.8 、 6.8$ 、 7.7 ; 每个位点的等位基因数从 2 到 11 个不等, 大小在 $110 \mathrm{bp}-259 \mathrm{bp}$ 之间. 位点 MFW 14 在 3 个群体中的等 位基因数最多, 分别为 $11 、 10 、 11$, 位点 MFW19 在 3 个群体中的等位基因数最少, 均为 2 (表 2).

\section{表 23 个鲤鱼群体微卫星分析统计结果 "}

Tab. 2 Summary of statistics for three populations of Cyprinus carpio L. with microsatellite analysis

\begin{tabular}{|c|c|c|c|c|c|c|c|c|c|c|c|c|c|c|c|c|}
\hline \multirow{2}{*}{ 微卫星位点 } & \multicolumn{5}{|c|}{ DP } & \multicolumn{5}{|c|}{$\mathrm{WS}$} & \multicolumn{6}{|c|}{$\mathrm{SY}$} \\
\hline & $\overline{N_{A}}$ & $H_{o}$ & $H_{E}$ & $P I C$ & $\overline{\mathrm{HW}}$ & $N_{A}$ & $H_{o}$ & $H_{E}$ & $P I C$ & $\overline{\mathrm{HW}}$ & $N_{A}$ & $H_{o}$ & $H_{E}$ & $P I C$ & HW & \\
\hline HLJ010 & 5 & 0.7333 & 0.7079 & 0.6463 & NS & 5 & 0.9667 & 0.6938 & 0.6271 & $*$ & 5 & 0.7667 & 0.6825 & 0.6222 & $*$ & \\
\hline HLJ011 & 4 & 0.5667 & 0.6107 & 0.5322 & NS & 7 & 0.5000 & 0.8068 & 0.7664 & $* *$ & 7 & 0.7333 & 0.7972 & 0.7520 & $*$ & $*$ \\
\hline HLJ013 & 6 & 0.8000 & 0.8023 & 0.7578 & $* *$ & 7 & 0.7333 & 0.8497 & 0.8137 & $* *$ & 5 & 0.7667 & 0.7243 & 0.6600 & NS & \\
\hline MFW1 & 6 & 1.0000 & 0.7051 & 0.6627 & $*$ & 8 & 1.0000 & 0.7379 & 0.6937 & $*$ & 9 & 1.0000 & 0.7243 & 0.6853 & $*$ & \\
\hline MFW3 & 8 & 1.0000 & 0.7932 & 0.7529 & NS & 9 & 0.9654 & 0.8512 & 0.7031 & NS & 10 & 1.0000 & 0.8847 & 0.8563 & $*$ & $*$ \\
\hline MFW4 & 7 & 1.0000 & 0.6977 & 0.6516 & NS & 8 & 1.0000 & 0.8768 & 0.8466 & NS & 5 & 1.0000 & 0.6508 & 0.5714 & $*$ & $*$ \\
\hline MFW6 & 9 & 1.0000 & 0.8497 & 0.8192 & $* *$ & 7 & 0.8861 & 0.7621 & 0.6800 & $*$ & 10 & 1.0000 & 0.8616 & 0.8306 & $*$ & $*$ \\
\hline MFW 13 & 4 & 1.0000 & 0.7441 & 0.6821 & $*$ & 5 & 1.0000 & 0.7023 & 0.6370 & $*$ & 5 & 1.0000 & 0.7271 & 0.6652 & $*$ & $*$ \\
\hline MFW14 & 11 & 1.0000 & 0.8949 & 0.8682 & $* *$ & 10 & 1.0000 & 0.8831 & 0.8545 & $*$ & 11 & 1.0000 & 0.8989 & 0.8729 & $*$ & $*$ \\
\hline MFW16 & 9 & 1.0000 & 0.8684 & 0.8373 & $* *$ & 9 & 1.0000 & 0.8881 & 0.8601 & $* *$ & 10 & 1.0000 & 0.8893 & 0.8614 & $*$ & $*$ \\
\hline MFW18 & 4 & 1.0000 & 0.6831 & 0.6019 & $*$ & 6 & 0.9751 & 0.8365 & 0.6431 & $*$ & 8 & 1.0000 & 0.8486 & 0.4857 & $*$ & $*$ \\
\hline MFW19 & 2 & 0.6667 & 0.4520 & 0.3457 & $* *$ & 2 & 0.3000 & 0.2593 & 0.2225 & $\mathrm{NS}$ & 2 & 0.5600 & 0.3814 & 0.3047 & NS & S \\
\hline MFW30 & 4 & 0.1667 & 0.4616 & 0.4199 & $* *$ & 5 & 0.6333 & 0.5921 & 0.5189 & $* *$ & 6 & 0.1667 & 0.7780 & 0.7337 & $*$ & $*$ \\
\hline 平均值 & 6.8 & 0.8410 & 0.7131 & 0.6598 & & 6.8 & 0.8430 & 0.7492 & 0.6820 & & 7.7 & 0.8456 & 0.7576 & 0.6847 & & \\
\hline
\end{tabular}

$* N_{A}$ : 等位基因数; $H_{O}$ : 杂合度观测值; $H_{E}$ : 杂合度期望值; $P I C$ : 多态信息含量 $; \mathrm{HW}$ : 哈代 - 温伯格平 衡; NS: 非显著水平 $(P>0.05) ; *$ : 显著水平 $(P<0.05) ; * *$ : 极显著水平 $(P<0.01)$

\section{2 遗传杂合度和多态信息含量}

3 个群体的平均观测杂合度和平均期望杂合度分别为 $0.8432 、 0.7400$, 其中 SY 群体最高, WS 群体次 之,DP群体最低. 位点 MFW1、MFW4、MFW13、MFW14、MFW16 在 3 个群体中的观测杂合度均达到了 1.0000. 位点 MFW30 在 DP 群体和 SY 群体中的观测杂合度最低,均为 0.1667 (表 2).

从表 2 的数据可以看出, PIC 最高的为 DP 群体的 MFW14 位点 $(0.8682)$, 最低的为 WS 群体的 MFW19 位点 $(0.2225) .3$ 个群体的平均 PIC 为 0.6755 , 其中 DP 群体为 0.6598 , WS 群体为 $0.6820, \mathrm{SY}$ 群体为 0.6847 .

\section{3 哈代 - 温伯格平衡和群体遗传分化}

3 个鲤鱼群体中, 共有 9 个位点符合哈代 - 温伯格平衡, 其余 30 个位点均偏离了哈代 - 温伯格平衡. 位点 MFW1、MFW6、MFW13、MFW14、MFW16、MFW18、MFW30 在所有群体中均偏离了哈代 - 温伯格平衡 (表 2). 从表 3 的数据可知, 3 个群体的 Shannon 多样性指数分别为 $1.5688 、 1.5603 、 1.6947$, 平均为 1.6079 . 平均 Fis 值均小于 0 , 分别为 $-0.1862 、-0.1279 、-0.1441$, 这表明 3 个鲤鱼群体均存在观测杂合度过剩的 现象. 
表 33 个鲤鱼群体的 Fis 值及 Shannon 多样性指数 $(I)$

Tab. 3 Fis value and Shannon genetic diversity index $(I)$ among three populations of Cyprinus carpio

\begin{tabular}{cccc}
\hline 参数 & DP & WS & SY \\
\hline Fis & -0.1862 & -0.1279 & -0.1441 \\
I & 1.5688 & 1.5603 & 1.6947 \\
\hline
\end{tabular}

表 43 个鲤鱼群体间的遗传相似性指数及遗传距离 *

Tab. 4 Genetic similarity indices and genetic distances among three populations of Cyprinus carpio L.

\begin{tabular}{cccc}
\hline 群体 & DP & WS & SY \\
\hline DP & 1 & 0.8288 & 0.8315 \\
WS & 0.1712 & 1 & 0.8320 \\
SY & 0.1685 & 0.1680 & 1 \\
\hline
\end{tabular}

* 表中上三角中的数字表示遗传相似性指数, 下三角中的数字表示遗传距离

\begin{tabular}{|c|c|c|c|}
\hline 0.200 & 0.150 & 0.100 & 0.050 \\
\hline
\end{tabular}

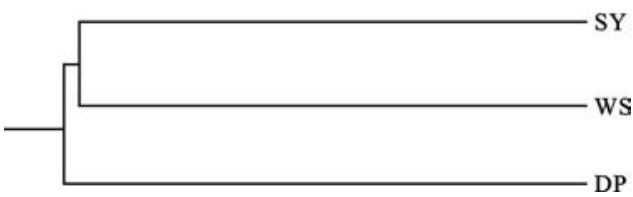

图 1 基于 Nei' $\mathrm{s}$ 遗传距离构建的 3 个鲤鱼群体 UPGMA 树

Fig. 1 UPGMA dendrogram based on Nei's genetic distance for three populations of Cyprinus carpio

\section{4 群体间的亲缘关系}

根据 Nei 指数法 ${ }^{[16]}$ 计算 3 个鲤鱼群体之间的 遗传相似性指数和遗传距离 (表 4). WS 群体与 SY 群体间的遗传相似系数最大 $(0.8320)$, 遗传距离 最小 $(0.1680) ; \mathrm{WS}$ 群体与 DP 群体间的遗传相似 系数最小 $(0.8288)$, 遗传距离最大 $(0.1712)$. 图 1 是基于 Nei's 遗传距离构建的 UPGMA 树, 3 个鲤 鱼群体聚成两支,其中 WS 群体与 SY 群体聚成一 支, DP 群体单独一支.

\section{3 讨论}

\section{1 鲤鱼群体的高度多态性}

一般而言, 1 对微卫星特异引物在纯合的材料 中只能扩增出 1 条 DNA 条带 ${ }^{[17]}$. 本研究中, 位点 MFW 1、MFW4、和 HLJ011 (图 2) 等在鲤鱼 3 个群体 的某些个体中扩增出了 $3-6$ 条带, 且位点 MFW 1、 MFW 14 在所有个体中均产生两个扩增位点,这可 能与鲤鱼是种间杂交的 4 倍体有关. 李建中等 ${ }^{[18]}$ 利用 15 对微卫星引物研究了异源四倍体鲫鲤及其 原始亲本红鲫和野鲤,结果发现有 14 对引物在 3 种鱼共 12 个个体中检测到了 $2-8$ 个等位基因. 刘 静霞等 ${ }^{[19]}$ 在镜鲤的 4 个人工雌核发育家系的一些 个体中也发现了类似的情况. 本研究中, 3 个鲤鱼 群体的平均观测杂合度值在 $0.8410-0.8456$ 之 间,平均期望杂合度值在 $0.7131-0.7576$ 之间, 遗 传杂合度水平均高于黄河鲤 ${ }^{[5]}$ 、长江鲤 ${ }^{[12]}$ 和匈牙 利的 6 个鲤鱼群体 ${ }^{[20]}$, 表现出了较高的遗传变异 水平. 3 个鲤鱼群体的平均 PIC 为 0.6755 ,其中 $\mathrm{SY}$ 群体最高 $(0.6847), W S$ 群体次之 $(0.6820)$, DP 群 体最低 $(0.6598)$. 可见, 3 个鲤鱼群体具有丰富的 遗传多样性,水质污染和过度捕捞等不良因素, 还

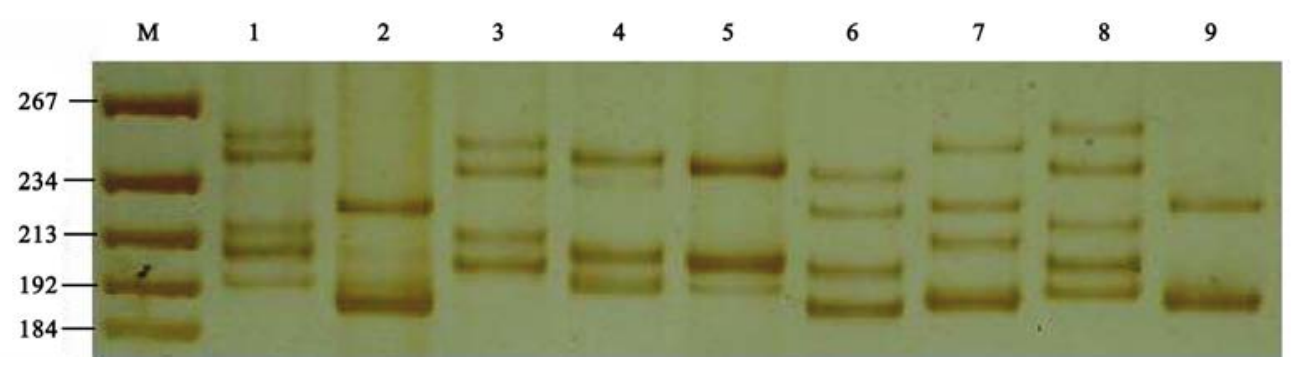

图 2 鲤鱼在微卫星位点 HLJ011 的电泳图

( M : DNA 分子量标记 pBR322/BsuRI; M : pBR322/BsuRI marker)

Fig. 2 Electrophoresis of microsatellite locus HLJ011 in Cyprinus carpio L. 
未对鲤鱼造成严重的危害, 但有关部门仍需提高警惕, 及时制定并严格执行鱼类资源保护及合理利用的相 关规章制度.

\section{2 哈代 - 温伯格平衡}

当群体处于哈代 - 温伯格平衡时,各等位基因的分布频率应该是相对稳定的,观察杂合度和期望杂合 度之间没有显著的差异 ${ }^{[21]}$. Istvan 等 $^{[20]}$ 利用 12 个微卫星标记研究了匈牙利 6 个鲤鱼品种的遗传结构, 结 果表明有一半以上的位点在野生鲤鱼品种 (Dunai) 中偏离了哈代 - 温伯格平衡. 本研究中, 3 个鲤鱼群体在 绝大多数位点呈现杂合过剩现象 (Fis < 0, 表 3), 只有一个位点 (HLJ011) 例外, 并且大多数位点 (77\%) 偏离 了哈代 - 温伯格平衡 (表 2). 这一方面可能是由于在一些位点存在无效等位基因, 而我们却把这种情况当 作杂合子处理, 从而引起基因型对哈代 - 温伯格平衡的偏离; 另一方面可能与 3 个群体鲤鱼与外来品种 (品 系) 发生了一定程度的杂交, 从而导致基因高度杂合有关. 从地理位置上看, 东平湖与黄河相通, 微山湖与 长江相通, 黄河鲤和长江鲤有可能分别进人两个湖区, 同原鲤鱼群体发生了基因交流, 逃逸的围网养殖品种 也有可能与原鲤鱼群体发生基因交流; 而 SY 群体本身为杂交鲤, 因此,这可能是导致 3 个鲤鱼群体大量微 卫星位点偏离哈代 - 温伯格平衡的主要原因.

\section{3 群体间的亲缘关系}

遗传相似性指数和遗传距离是衡量群体间亲缘关系的重要的指标. 一级亲缘关系的个体间遗传相似指 数为 0.5 , 二级亲缘关系为 $0.25^{[22]}$. ZHOU 等 ${ }^{[6]}$ 研究表明,长江鲤与兴国红鲤间的遗传相似指数为 0.9549 , 而与俄罗斯镜鲤间的遗传相似指数仅为 0.0538 ,说明长江鲤与兴国红鲤间的遗传差异较小,群体分化时间 不长; 相反,与俄罗斯镜鲤间的遗传差异较大,群体分化时间较长. 微山湖是中国北方第一大淡水湖泊, 京杭 大运河贯穿其南北, 因此长江水系鲤鱼能够沿着大运河进入微山湖, 从而使微山湖鲤鱼携带了长江鲤的基 因. 泰安市水产研究所是重要的鲤鱼良种供应基地,为了保证商品鱼对环境的适应能力和快速生长,该所每 年都从长江水系引人优质的雄性亲鱼进行繁殖, 因此 SY 鲤鱼群体也携带长江鲤的基因. 研究结果表明, 这 两个群体鲤鱼亲缘关系最近,遗传距离最小 $(0.1680)$.

\section{4 参考文献}

[1] 刘明华,沈俊宝,白庆利等. 新品种高寒鲤的选育. 水产学报,1997,21(4): $391-397$.

[2] 沈俊宝, 刘明华. 荷包红鲤抗寒品系的篮选. 淡水渔业, 1988,3:314-317.

[3] 孙小异,张建森,施永红等. 建鲤遗传特性的研究. 水产学报, 1994,18(3): $205-213$.

[4] 杜长斌,孙效文,楼允东等. 应用微卫星技术对野鲤和两种鲤选育品系的遗传多样性分析. 上海水产 大学学报,2000,9(4): 285-289.

[5]常玉梅,孙效文,梁立群. 中国鲤几个代表种群基因组 DNA 遗传多样性分析. 水产学报, 2004,28 (5) : $481-486$.

[6] Zhou J, Wu Q, Wang Z, et al. Genetic variation analysis within and among six varieties of common carp (Cyprinus carpio L.) in china using microsatellite markers. Russian Journal of Genetics, 2004,40 (10): $1144-1148$.

[7] Desvignes J F, Laroche J, Durand J D, et al. Genetic variability in reared stocks of common Carp( Cyprinus carpio L. ) based on allozymes and microsatellites. Aquaculture, 2001,194: $291-301$.

[8] Rana R S, Bhat K V, Lakhanpal S, et al. Comparative genetic diversity in natural and hatchery populations of Indian major carp(C. catla and L. rohita). Asian-Aust J Anim Sci, 2004,17(9): 1197 - 1203.

[9] Dietrich W, Katz H, Linclon S E. A genetic map of the mouse suitable for typing intraspecific crosses. Genetics $, 1992,131: 423-447$

[10] 孙效文,梁利群. 鲤鱼的遗传连锁图谱. 中国水产科学, 2000,7(1): $1-5$.

[11］董 仕,谷口顺彦. 微卫星 DNA 鉴别克隆香鱼. 水产学报, 2003,27(4): 295 - 299.

[12] Roques S, Sevigny J M, Bernatchez. Genetic structure of deep-water redfish, Sebastes mentella, populations across the North Atlantic. Marine Biology,2002,140:297 - 307 . 
[13] 马洪雨,姜运良, 郭金峰等. 利用微卫星标记分析东平湖黄颡鱼的遗传多样性. 激光生物学报, 2006, 15(2).

[14] 程 超, 明庆磊. 微山湖鲤鱼生理生化血液流变学性质的研究. 江苏农业科学, 2005, (1): $95-96$.

[15] 马洪雨, 姜运良, 岳永生. 一种从鱼类肌肉组织中提取基因组 DNA 的简易方法. 生物技术通讯, $2005, \mathbf{1 6}(5): 531-532$.

[16] Nei M. Estimation of average heterozygosity and genetic distance from a small number of individuals. Genetics, $1978,89: 583-590$.

[17] Crooijmans R P M A, Bierbooms V A F, Komen J, et al. Microsatellite markers in common carp (Cyprinus carpio L. ). Animal Genetics, $1997,28: 129$ - 134.

[18] 李建中, 刘少军, 张轩杰等. 异源四倍体鲫鲤及其原始亲本遗传变异的微卫星标记分析. 遗传学报, 2005,32(4) : $378-383$.

[19] 刘静霞, 周 莉, 赵振山等. 锦鲤 4 个人工雌核发育家系的微卫星标记研究. 动物学研究, 2002,23 (2) :97- 105 .

[20] Istvan L, Istvan M, Csaba H, et al. Preliminary studies on the genetic variability of six Hungarian common carp strains using microsatellite DNA markers. Hydrobiologia, 2005,533:223-228.

[21] 常 青, 曹发华, 朱立峰等. 长江下游地区夜鹗种群微卫星变异及遗传多样性. 动物学报, 2005,51 (4) :657-663.

[22] 梁立群,孙效文,董崇智等. 五种鲟、鳇鱼基因组 DNA 遗传多样性分析. 中国水产科学, 2002,9(3): $273-276$. 